\title{
Hemoglobinas de origem africana em comunidades quilombolas do estado do Tocantins, Brasil
}

\author{
Hemoglobins of African origin in quilombola communities of Tocantins State, Brazil \\ Annyelle Figueredo Teles, Luciana da Costa da Silva, Amanda Cordeiro da Silva, Lidiane Oliveira de Souza, Márcio \\ Galdino dos Santos, Carla Simone Seibert
}

Universidade Federal do Tocantins, Porto Nacional, Tocantins, Brasil

\begin{abstract}
RESUMO
OBJETIVO: Verificar a incidência de hemoglobinas de descendência africana ( $\mathrm{HbS}$ e $\mathrm{HbC}$ ) em comunidades quilombolas do estado do Tocantins, Brasil. MATERIAIS E MÉTODOS: Foi coletado o sangue de quilombolas em 14 comunidades do Estado; a triagem foi realizada em eletroforese de acetato de celulose ( $\mathrm{pH} 8,6$ ), e aquelas com padrão alterado foram submetidas à cromatografia líquida de alta eficiência, sendo registrados o gênero e a idade das pessoas amostradas. RESULTADOS: A análise dos resultados demonstrou que, dos 822 quilombolas investigados, 95 apresentaram hemoglobinas anormais, sendo 0,5\% com doença falciforme (HbSS); $5,7 \%$ traço para hemoglobina S (HbAS); 4,9\% traço para hemoglobina $\mathrm{C}(\mathrm{HbAC}) ; 0,2 \%$ com hemoglobina fetal aumentada; $0,1 \%$ com hemoglobina $A_{2}$ aumentada; e $88,4 \%$ com hemoglobina normal (HbAA). HbSS foi observada na faixa etária infantil e adolescente e HbAS e $\mathrm{HbAC}$ em todas as faixas etárias. Em relação ao sexo, não foi possível sugerir o efeito materno para $\mathrm{HbS}$, devido ao maior quantitativo de pessoas do sexo masculino com essa informação genética. CONCLUSÃO: Neste estudo, a incidência das $\mathrm{HbS}$ e HbC, observada nas comunidades quilombolas, esteve dentro do esperado para a Região Norte do Brasil. No entanto, destacam-se a elevada prevalência da doença falciforme e a grande frequência de traço falciforme em algumas das comunidades estudadas, com atenção especial para a região sul do Estado. Desse modo, os resultados aqui apresentados sinalizam risco iminente para o aumento da incidência da doença no Tocantins.
\end{abstract}

Palavras-chave: Anemia Falciforme; Traço Falciforme; Hemoglobina C; Hemoglobina Falciforme.

\begin{abstract}
OBJECTIVE: To verify the incidence of hemoglobins of African descent ( $\mathrm{HbS}$ and $\mathrm{HbC}$ ) in quilombola communities in Tocantins State, Brazil. MATERIALS AND METHODS: Blood samples were collected from 14 quilombola

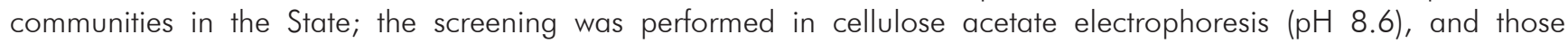
samples with altered standard were submitted to high performance liquid chromatography, recording gender and age of the quilombola people. RESULTS: The analysis of the results showed that of the 822 quilombolas investigated 95 presented abnormal hemoglobins, $0.5 \%$ with sickle cell disease (HbSS); $5.7 \%$ for hemoglobin $\mathrm{S}$ trait $(\mathrm{HbAS}) ; 4.9 \%$ for hemoglobin $\mathrm{C}$ trait $(\mathrm{HbAC}) ; 0.2 \%$ with increased fetal hemoglobin; $0.1 \%$ with increased hemoglobin $\mathrm{A}_{2}$; and $88.4 \%$ with normal hemoglobin ( $\left.\mathrm{HbAA}\right)$. HbSS was observed in infant and adolescent groups and $\mathrm{HbAS}$ and $\mathrm{HbAC}$ in all age groups. About sex, it was not possible to suggest the maternal effect for $\mathrm{HbS}$ due to the greater quantitative of males with this genetic information. CONCLUSION: In the current study the incidence of $\mathrm{HbS}$ and $\mathrm{HbC}$ observed in quilombola communities was within the expected range for the Northern Region of Brazil. However, the high prevalence of sickle cell disease and the high frequency of sickle cell trait were highlighted in some quilombola communities with special attention to the southern region of the State. Thus, the results presented in this study indicate an imminent risk for the increased incidence of that disease in Tocantins.
\end{abstract}

Keywords: Sickle Cell Anemia; Sickle Cell Trait; Hemoglobin C; Sickle Hemoglobin.

\footnotetext{
Correspondência / Correspondence:

Carla Simone Seibert

Rua 03, lote 17, s/n. Bairro: Jardim dos Ipês - CEP: 77500-000 - Porto Nacional, Tocantins, Brasil - Tel.: +55 (63) $3363-9434$

E-mail: seibertcs@uft.edu.br
} 


\section{INTRODUÇÃO}

Várias alterações no funcionamento dos eritrócitos e estrutura química das hemoglobinas surgiram em decorrência de pressões seletivas contra os efeitos letais das infecções causadas por Plasmodium spp. (malária) observadas em regiões da África, Índia e Ásia. $\mathrm{Na}$ África, as hemoglobinas variantes mais frequentes são a hemoglobina $\mathrm{S}(\mathrm{HbS})$ e a hemoglobina C $(\mathrm{HbC})$, que se distribuem de forma heterogênea nas diversas regiões do continente ${ }^{1,2}$. Ser portador dessas hemoglobinas representa um fator adaptativo importante nos locais onde a malária é endêmica, pois garante maior resistência à infecção causada pelo parasita, o que significa maior proteção contra a doença ${ }^{3,4}$.

Quando as hemoglobinas variantes são herdadas dos dois genitores (pai e mãe), na forma homozigota ( $\mathrm{HbSS}$ ou $\mathrm{HbCC}$ ), dupla heterozigota ( $\mathrm{HbSC})$ ou associada com outras hemoglobinopatias ( $\mathrm{HbS} / \beta$-talassemias, $\mathrm{HbSD}$; $\mathrm{HbSE}, \mathrm{HbC} / \beta$-talassemias, entre outras), a pessoa pode apresentar alterações clínicas graves. O conjunto de manifestações clínicas desencadeadas por essas associações é denominado de doença falciforme $(D F)^{5}$. Essa é uma das doenças hereditárias mais prevalentes no mundo e sua distribuição afeta principalmente os negros da África e da América do Norte, bem como as populações latinas ${ }^{6}$.

No Brasil, a inserção da HbS e da HbC está relacionada com a introdução de indivíduos de inúmeras tribos africanas, iniciada em 1550, para o trabalho escravo. O fluxo migratório dessa população se expandiu para várias regiões do país após a abolição da escravatura, iniciando uma pan-mixia racial que hoje é uma característica do Brasil ${ }^{7}$. Essa composição afrodescendente da população brasileira leva a DF a integrar um grupo de doenças de agravos relevantes e, por essa razão, foi incluída nas ações da Política Nacional de Atenção Integral à Saúde da População Negra, do Ministério da Saúde ${ }^{8}$.

A forma mais prevalente da DF no Brasil é a homozigose da HbS (HbSS - anemia falciforme). Sua natureza molecular é a substituição de uma valina por um ácido glutâmico (GAG $\rightarrow$ GTG) na sexta posição da cadeia betaglobina. As moléculas de $\mathrm{HbS}$ se organizam em longos polímeros, formando "cristais" intraeritrocitários, o que ocasiona a deformação das células e a forma de foice característica da doença, $a^{9,10}$

$\mathrm{Na} \mathrm{HbC} \mathrm{ocorre} \mathrm{a} \mathrm{substituição} \mathrm{do} \mathrm{ácido} \mathrm{glutâmico}$ por uma lisina, também na sexta posição da cadeia betaglobina. É uma hemoglobina de agregação que também forma "cristais" intraeritrocitários, porém em menor frequência e intensidade que a HbS, sendo a segunda hemoglobina variante mais frequente entre os brasileiros ${ }^{11,12}$.

A pessoa com DF pode apresentar mais de $90 \%$ de $\mathrm{HbS}$ em seus eritrócitos. A polimerização intracelular ocasionada pela $\mathrm{HbS}$ propicia a perda de água e potássio, levando à formação de células mais densas, rígidas e de pouca deformabilidade, o que é responsável pelas principais manifestações clínicas da doença: hemólise e vaso-oclusão. Além de episódio de dor, a hipóxia tecidual, que é decorrente da oclusão vascular, ocasiona danos isquêmicos e perda progressiva da função de órgãos e tecidos ${ }^{10,13,14}$.

Uma das particularidades da DF é a sua variabilidade clínica, caracterizando-se por diferentes graus de anemia hemolítica. Dessa maneira, enquanto alguns pacientes têm um quadro de grande gravidade e estão sujeitos a inúmeras complicações e frequentes hospitalizações, outros podem apresentar uma evolução mais benigna, em alguns casos quase assintomática. A sua variabilidade clínica é expressa por fatores genéticos relacionados ao nível de hemoglobina fetal, associação com as talassemias e os haplótipos. No entanto, outros fatores podem ser relacionados à variabilidade clínica da doença, como condição socioeconômica, qualidade alimentar, prevenção de infecções e assistência médica ${ }^{15,16}$.

As pessoas que apresentam a informação parcial para essas hemoglobinas variantes (HbAS ou HbAC, também denominado traço falciforme) não manifestam nenhum dos sintomas clínicos da DF. No entanto, alguns pesquisadores associam as complicações clínicas observadas em alguns portadores de traço falciforme às condições que propiciam o processo de falcização dos eritrócitos, especialmente quando são submetidos à baixa tensão de oxigênio, acidose e desidratação ${ }^{17}$.

Estudos da prevalência de pessoas com $\mathrm{HbS}$ e $\mathrm{HbC}$ geralmente são realizados em populações de diferentes origens étnicas ${ }^{18,19,20}$, sendo pouco desenvolvidos em comunidades quilombolas ${ }^{21,22,23}$. Atualmente, no Brasil, há 2.431 comunidades quilombolas em 24 estados brasileiros, estando o Tocantins com 38 comunidades certificadas pela Fundação Cultural Palmares ${ }^{24}$. São comunidades formadas por remanescentes de quilombos, que vivem isolados, cultural e geograficamente, e que necessitam de atenção às doenças do seu grupo étnico-racial. Dessa forma, o objetivo do presente estudo foi verificar a incidência de DF e, consequentemente, de hemoglobinas de origem africana em comunidades quilombolas do Tocantins.

\section{MATERIAIS E MÉTODOS}

Este estudo foi realizado em 14 comunidades quilombolas do estado do Tocantins: Santa Maria das Mangueiras, no município de Dois Irmãos; Mumbuca e arredores; Carrapato, Formiga e Ambrósio; e Quilombolas dos Rios, no município de Mateiros; Chapada de Natividade e São José, no município de Chapada de Natividade; Redenção, no município de Natividade; Lagoa da Pedra e Kalunga do Mimoso, no município de Arraias; Laginha e áreas vizinhas e São Joaquim, no município de Porto Alegre do Tocantins; Lajeado, no município de Dianópolis; Baião e Poço Dantas, no município de Almas. 
Inicialmente, os representantes das comunidades quilombolas foram informados do objetivo do estudo e questionados acerca do interesse em participar da pesquisa. Quando a resposta era afirmativa, seguia-se o agendamento da visita à comunidade. Nas comunidades, as famílias foram esclarecidas sobre a DF e dos procedimentos adotados na pesquisa.

As pessoas que participaram do estudo assinaram o Termo de Consentimento Livre e Esclarecido, conforme a Resolução n 196/96, do Conselho Nacional de Saúde, e os menores de idade, somente quando autorizados pelos pais ou outro responsável. $\bigcirc$ presente trabalho foi autorizado pelo Comitê de Ética em Pesquisa da Universidade Federal do Tocantins, processo $n^{\circ}$ 048/2009, em 30 de abril de 2010. As visitas às comunidades ocorreram de outubro de 2011 a outubro de 2012.

A coleta de sangue para o diagnóstico das hemoglobinas foi realizada por punção venosa (cerca de $3 \mathrm{~mL}$ ); as amostras foram acondicionadas em tubos, com etilenodiamino tetracético (EDTA 10\%). A triagem das amostras foi realizada em eletroforese de acetato de celulose (pH 8,6), e aquelas com mobilidade eletroforética alterada foram submetidas à cromatografia líquida de alta eficiência ${ }^{25}$, a qual possibilitou identificar, com exatidão, os tipos de hemoglobina e o seu percentual.

Foi registrado o número de pessoas por família, assim como a faixa etária e o gênero de cada pessoa cujo sangue foi coletado, com a finalidade de também tabular os dados. As informações da faixa etária foram organizadas em cinco categorias: criança (0 a 12 anos incompletos), adolescente (12 a 18 anos incompletos), jovem (18 a 29 anos incompletos), adulto (29 a 60 anos incompletos) e idoso (acima de 60 anos), tendo como base o Estatuto da Criança e do Adolescente ${ }^{26}$, Estatuto da Juventude ${ }^{27}$ e Estatuto do Idoso ${ }^{28}$. Para a análise descritiva, os resultados foram apresentados em valores numéricos e percentagens.

\section{RESULTADOS}

Nas 14 comunidades quilombolas, foram visitadas 229 famílias. Foi realizada a investigação das hemoglobinas de 822 pessoas, o que representou 65\% de seus integrantes (Tabela 1). Não participaram da pesquisa as pessoas que não estavam na comunidade no dia da visita e aquelas que não tiveram interesse na investigação.

$\bigcirc$ resultado da investigação das hemoglobinas está apresentado na tabela 2. Das comunidades estudadas, duas não apresentaram pessoas com a informação genética para $\mathrm{HbS}$ ou $\mathrm{HbC}$, sendo essas Laginha e áreas vizinhas e Santa Maria das Mangueiras. $\mathrm{Na}$ comunidade Chapada de Natividade, foram encontradas quatro pessoas com DF e, nas demais comunidades, houve prevalência variada de traço para $\mathrm{HbS}$ e $\mathrm{HbC}$. Assim, das pessoas amostradas, 0,5\% apresentaram $\mathrm{HbS}$ em homozigose (HbSS - anemia falciforme), 5,7\% traço para $\mathrm{HbS}(\mathrm{HbAS}), 4,9 \%$ traço para $\mathrm{HbC}(\mathrm{HbAC}), 0,4 \% \mathrm{Hb}$ fetal aumentada $(\mathrm{HbF} \uparrow)$, $0,1 \% \mathrm{HbA}_{2}$ aumentada $\left(\mathrm{HbA}_{2} \uparrow\right)$ e $88,4 \% \mathrm{HbAA}$ normal. Das pessoas com DF, três apresentaram concentração de $\mathrm{HbF}$ entre 8 e 10\%, e a outra, valor inferior a 3\%.

Em relação ao gênero, $48,5 \%$ das pessoas analisadas eram do gênero feminino e 51,5\% do masculino, o que revelou uma distribuição equilibrada entre os sexos (Tabela 3). As frequências de HbSS e traço para $\mathrm{HbC}$ foram maiores no gênero feminino $(0,4 \%$ e $2,7 \%$, respectivamente) e o traço para $\mathrm{HbS}$ prevaleceu no gênero masculino $(3,4 \%)$.

A faixa etária dos quilombolas que participaram deste trabalho está apresentada na tabela 4. Do total amostral, 37,4\% eram adultos, seguido de 24,3\% de crianças, $14,8 \%$ de adolescentes, $12,8 \%$ de idosos e $10,7 \%$ de jovens. A HbSS se concentrou na faixa etária das crianças $(0,4 \%)$ e adolescentes $(0,1 \%)$; contudo, o traço para $\mathrm{HbS}$ e $\mathrm{HbC}$ apareceu em todas as faixas etárias. A prevalência entre os adultos foi de $\operatorname{HbS}(2,6 \%)$.

Tabela 1 - Número de famílias das comunidades quilombolas, total de pessoas que integravam essas famílias, número e proporção daquelas que participaram da investigação do tipo de hemoglobinas no estado do Tocantins, Brasil, $2011-2012$

\begin{tabular}{lcccc}
\hline \multicolumn{1}{c}{ Comunidades } & \multirow{2}{*}{ Famílias } & Pessoas das famílias & Pessoas investigadas & N \\
\hline Baião & 13 & 55 & 40 & 72 \\
Carrapato, Formiga e Ambrósio & 45 & 218 & 141 & 65 \\
Chapada de Natividade & 23 & 109 & 61 & 56 \\
Kalunga do Mimoso & 75 & 243 & 188 & 77 \\
Laginha e áreas vizinhas & 12 & 79 & 47 & 59 \\
Lagoa da Pedra & 15 & 59 & 39 & 66 \\
Lajeado & 9 & 45 & 52 & 71 \\
Mumbuca e arredores & 24 & 108 & 29 & 50 \\
Poço Dantas & 15 & 52 & 29 & 56 \\
Quilombolas dos Rios & 14 & 70 & 45 & 41 \\
Redenção & 14 & 51 & 69 & 88 \\
Santa Maria das Mangueiras & 22 & 74 & 23 & 93 \\
São Joaquim & 10 & 49 & 25 & 47 \\
São José & 8 & 1.251 & 822 & 64 \\
\hline Total & 229 & & 65 \\
\hline
\end{tabular}


Tabela 2 - Número e proporção de pessoas, segundo os diferentes tipos de hemoglobinas, em 14 comunidades quilombolas do estado do Tocantins, Brasil, 2011-2012

\begin{tabular}{|c|c|c|c|c|c|c|c|c|c|c|c|c|c|c|}
\hline \multirow{3}{*}{ Comunidades } & \multicolumn{12}{|c|}{ Hemoglobinas* } & \multirow{2}{*}{\multicolumn{2}{|c|}{ Total $^{\dagger}$}} \\
\hline & \multicolumn{2}{|c|}{ SS } & \multicolumn{2}{|c|}{ AS } & \multicolumn{2}{|c|}{$A C$} & \multicolumn{2}{|c|}{$\mathrm{F} \uparrow$} & \multicolumn{2}{|c|}{$\mathrm{A}_{2} \uparrow$} & \multicolumn{2}{|c|}{ AA } & & \\
\hline & $\mathrm{N}$ & $\%$ & $\mathrm{~N}$ & $\%$ & $\mathrm{~N}$ & $\%$ & $\mathrm{~N}$ & $\%$ & $\mathrm{~N}$ & $\%$ & $\mathrm{~N}$ & $\%$ & $\mathrm{~N}$ & $\%$ \\
\hline Baião & - & - & 4 & 10,0 & - & - & - & - & - & - & 36 & 90,0 & 40 & 4,9 \\
\hline $\begin{array}{l}\text { Carrapato, Formiga e } \\
\text { Ambrósio }\end{array}$ & - & - & 3 & 2,1 & 12 & 8,5 & - & - & - & - & 126 & 89,4 & 141 & 17,2 \\
\hline Chapada de Natividade & 4 & 6,6 & 4 & 6,6 & 3 & 4,9 & - & - & - & - & 50 & 82,0 & 61 & 7,4 \\
\hline Kalunga do Mimoso & - & - & 17 & 9,0 & - & - & - & - & - & - & 171 & 91,0 & 188 & 22,9 \\
\hline Laginha e áreas vizinhas & - & - & - & - & - & - & - & - & - & - & 47 & 100,0 & 47 & 5,7 \\
\hline Lagoa da Pedra & - & - & 3 & 7,7 & - & - & - & - & - & - & 36 & 92,3 & 39 & 4,7 \\
\hline Lajeado & - & - & 2 & 6,3 & 8 & 25,0 & - & - & - & - & 22 & 68,8 & 32 & 3,9 \\
\hline Mumbuca e arredores & - & - & 2 & 3,7 & 3 & 5,6 & - & - & - & - & 49 & 90,7 & 54 & 6,6 \\
\hline Poço Dantas & - & - & 5 & 17,2 & 2 & 6,9 & - & - & - & - & 22 & 75,9 & 29 & 3,5 \\
\hline Quilombolas dos Rios & - & - & 3 & 10,3 & 2 & 6,9 & - & - & - & - & 24 & 82,8 & 29 & 3,5 \\
\hline Redenção & - & - & - & - & 1 & 2,2 & 3 & 6,7 & - & - & 41 & 91,1 & 45 & 5,5 \\
\hline $\begin{array}{l}\text { Santa Maria das } \\
\text { Mangueiras }\end{array}$ & - & - & - & - & - & - & - & - & - & - & 69 & 100,0 & 69 & 8,4 \\
\hline São Joaquim & - & - & 2 & 8,7 & 2 & 8,7 & - & - & - & - & 19 & 82,6 & 23 & 2,8 \\
\hline São José & - & - & 2 & 8,0 & 7 & 28,0 & - & - & 1 & 4,0 & 15 & 60,0 & 25 & 3,0 \\
\hline Total $^{+}$ & 4 & 0,5 & 47 & 5,7 & 40 & 4,9 & 3 & 0,4 & 1 & 0,1 & 727 & 88,4 & 822 & 100,0 \\
\hline
\end{tabular}

AA: Hemoglobina normal; SS: Anemia falciforme; AS: Traço para hemoglobina S; AC: Traço para hemoglobina C; $A_{2} \uparrow:$ Hemoglobina A aumentada; $\mathrm{F} \uparrow$ : Hemoglobina fetal aumentada; Sinal convencional utilizado: - Dado numérico igual a zero não resultante de arredondamento; * \%: Em relação ao total de cada comunidade; $+\%$ : Em relação ao total de todas as comunidades.

Tabela 3 - Número e proporção de gênero das pessoas de 14 comunidades quilombolas do estado do Tocantins, Brasil, que realizaram a investigação do tipo de hemoglobinas, 2011-2012

\begin{tabular}{|c|c|c|c|c|c|c|c|c|c|c|c|c|c|c|}
\hline & \multicolumn{12}{|c|}{ Hemoglobinas* } & \multirow{2}{*}{\multicolumn{2}{|c|}{ Total }} \\
\hline & \multicolumn{2}{|c|}{ SS } & \multicolumn{2}{|c|}{ AS } & \multicolumn{2}{|c|}{$A C$} & \multicolumn{2}{|c|}{$\mathrm{F} \uparrow$} & \multicolumn{2}{|c|}{$\mathrm{A}_{2} \uparrow$} & \multicolumn{2}{|c|}{ AA } & & \\
\hline & $\mathrm{N}$ & $\%$ & $\mathrm{~N}$ & $\%$ & $\mathrm{~N}$ & $\%$ & $\mathrm{~N}$ & $\%$ & $\mathrm{~N}$ & $\%$ & $\mathrm{~N}$ & $\%$ & $\mathrm{~N}$ & $\%$ \\
\hline Feminino & 3 & 0,4 & 19 & 2,3 & 22 & 2,7 & 3 & 0,4 & - & - & 352 & 42,8 & 399 & 48,5 \\
\hline Masculino & 1 & 0,1 & 28 & 3,4 & 18 & 2,2 & - & - & 1 & 0,1 & 375 & 45,6 & 423 & 51,5 \\
\hline Total & 4 & 0,5 & 47 & 5,8 & 40 & 4,9 & 3 & 0,4 & 1 & 0,1 & 727 & 88,4 & 822 & 100,0 \\
\hline
\end{tabular}

AA: Hemoglobina normal; SS: Anemia falciforme; AS: Traço para hemoglobina S; AC: Traço para hemoglobina $C_{;} A_{2} \uparrow$ : Hemoglobina $A_{2}$ aumentada; $\mathrm{F} \uparrow$ : Hemoglobina fetal aumentada; Sinal convencional utilizado: - Dado numérico igual a zero não resultante de arredondamento; ${ }^{*} \%$ : Em relação ao total dos dois gêneros.

Tabela 4 - Número e proporção da faixa etária das pessoas de 14 comunidades quilombolas do estado do Tocantins, Brasil, que participaram da investigação do tipo de hemoglobinas, 2011-2012

\begin{tabular}{|c|c|c|c|c|c|c|c|c|c|c|c|c|c|c|}
\hline \multirow{3}{*}{ Faixa etária } & \multicolumn{12}{|c|}{ Hemoglobinas* } & \multirow{2}{*}{\multicolumn{2}{|c|}{ Total }} \\
\hline & \multicolumn{2}{|c|}{ SS } & \multicolumn{2}{|c|}{ AS } & \multicolumn{2}{|c|}{$A C$} & \multicolumn{2}{|c|}{$\mathrm{F} \uparrow$} & \multicolumn{2}{|c|}{$\mathrm{A}_{2} \uparrow$} & \multicolumn{2}{|c|}{$\mathrm{AA}$} & & \\
\hline & $\mathrm{N}$ & $\%$ & $\mathrm{~N}$ & $\%$ & $\mathrm{~N}$ & $\%$ & $\mathrm{~N}$ & $\%$ & $\mathrm{~N}$ & $\%$ & $\mathrm{~N}$ & $\%$ & $\mathrm{~N}$ & $\%$ \\
\hline Criança & 3 & 0,4 & 10 & 1,2 & 11 & 1,3 & - & - & - & - & 176 & 21,4 & 200 & 24,3 \\
\hline Adolescente & 1 & 0,1 & 3 & 0,4 & 11 & 1,3 & 3 & 0,4 & - & - & 104 & 12,7 & 122 & 14,8 \\
\hline Jovem & - & - & 6 & 0,7 & 7 & 0,9 & - & - & - & - & 75 & 9,1 & 88 & 10,7 \\
\hline Adulto & - & - & 21 & 2,6 & 7 & 0,9 & - & - & 1 & 0,1 & 278 & 33,8 & 307 & 37,4 \\
\hline Idoso & - & - & 7 & 0,9 & 4 & 0,5 & - & - & - & - & 94 & 11,4 & 105 & 12,8 \\
\hline Total & 4 & 0,5 & 47 & 5,8 & 40 & 4,9 & 3 & 0,4 & 1 & 0,1 & 727 & 88,4 & 822 & 100,0 \\
\hline
\end{tabular}

AA: Hemoglobina normal; SS: Anemia falciforme; AS: Traço para hemoglobina S; AC: Traço para hemoglobina C; $A_{2} \uparrow$ : Hemoglobina A aumentada; $\mathrm{F} \uparrow$ : Hemoglobina fetal aumentada; Sinal convencional utilizado: - Dado numérico igual a zero não resultante de arredondamento; ${ }^{*} \%$ : Em relação ao total de todas as faixas etárias. 


\section{DISCUSSÃO}

Foram encontradas quatro pessoas com DF dentre as 822 analisadas $(1: 205)$. A prevalência observada neste estudo foi superior à estimada pelo Ministério da Saúde (1:1.000 para recém-nascidos vivos) e a constatada na Bahia (1:650 pessoas), Estado com - maior número de doentes diagnosticados até 0 momento ${ }^{14,29}$

As pessoas com DF pertenciam a duas famílias com laços de parentesco, ambas da comunidade Chapada de Natividade. Em uma das famílias, três irmãos portavam a DF, sendo um menino de 5 anos de idade, uma menina de 10, e uma adolescente de 15; na outra, uma menina de 5 anos. Estudos destacaram que, há mais de 20 anos, o diagnóstico tardio da DF é um importante fator em relação a óbitos em pacientes jovens. Esse fator somado à desorientação familiar diante das complicações da doença, à falta de medicamentos nos postos de saúde e à falta de atendimento ambulatorial rigoroso contribuem para o óbito em doentes de pouca idade ${ }^{30,31}$.

A concentração de HbF nas pessoas diagnosticadas com DF foi de 3 a 10\%. Comparando esses dados com a literatura, observa-se uma tendência para que sejam haplótipos do tipo CAR ou Benin, que são os mais frequentes na população brasileira, o que precisaria ser confirmado com estudos de biologia molecular. Os níveis de hemoglobina fetal e os haplótipos do cluster do gene da betaglobina têm sido considerados como fatores determinantes da variabilidade da DF. $O$ haplótipo CAR (República Centro-Africana) apresenta níveis mais baixos de hemoglobina fetal $(<5 \%)$ e curso clínico mais grave da doença, enquanto que o Benin tem níveis de hemoglobina fetal entre 5 e 15\%, com curso clínico moderado ${ }^{15,32}$.

Relatos de óbitos de pessoas diagnosticadas com DF na comunidade de Carrapato, Formiga e Ambrósio, localizada no município de Mateiros, foram obtidos durante o desenvolvimento deste trabalho. Relatos de óbitos pela DF também foram registrados em outros trabalhos realizados em comunidades quilombolas do Tocantins ${ }^{22,23}$. É importante destacar que, somente nas comunidades com histórico de pessoas com DF, a população relatou conhecer alguns aspectos da doença; caso contrário, o assunto foi praticamente desconhecido. A falta de conhecimento sobre a DF é um fator de risco que aumenta a probabilidade de novos doentes, principalmente em comunidades quilombolas, onde há maior isolamento geográfico e difícil acesso à informação e ao diagnóstico.

Os resultados deste trabalho mostraram que a incidência do traço para $\mathrm{HbS}$ nas comunidades quilombolas do Tocantins foi de 1:17 pessoas, dado que se igualou ao encontrado na Bahia, sendo superior ao observado nos estados do Rio de Janeiro (1:21), Pernambuco (1:23) e Minas Gerais (1:23). No Brasil, a incidência do traço para $\mathrm{HbS}$ é de 1:35 nascidos vivos, e estima-se o nascimento de 200.000 crianças com essa informação genética ${ }^{14}$.
A prevalência do traço para $\mathrm{HbS}$ nas regiões do Brasil é de 6 a 10\% para Norte e Nordeste, e de 2 a 3\% para Sul e Sudeste ${ }^{14}$. Assim, a frequência obtida para as comunidades quilombolas do Tocantins $(5,7 \%)$ mostrou-se dentro do esperado para o norte do país. No entanto, duas das comunidades analisadas neste trabalho chamaram a atenção para a frequência de traço para HbS, sendo essas Poço Dantas $(17,2 \%)$ e Quilombolas dos Rios (10,3\%). Estudos realizados na comunidade Malhadinha, município de Brejinho de Nazaré, e na Distrito do Morro de São João, município de Santa Rosa, ambas localizadas no Tocantins, também revelaram incidência preocupante de pessoas com traço falciforme $112,5 \%$ e $14,6 \%$, respectivamente $)^{20,21}$. $\bigcirc$ elevado percentual de $\mathrm{HbS}$ dessas comunidades se aproxima do observado para alguns países do continente africano: em Gana, ele varia de 10 a 22\% e, em Angola, de 11 a 37\%. Tal semelhança com os países africanos pode ser explicada pela história de origem da população brasileira. Estudos de DNA mitocondrial das pessoas com DF revelaram que a maioria dos negros do Brasil é originária de regiões da África Central, como Angola, Benin, Gana e Togo 29,33 .

Não foram detectados indivíduos com $\mathrm{HbC}$ em homozigose $(\mathrm{HbCC})$, contudo a frequência de traço para $\mathrm{HbC}$ foi de 4,9\% (1:20), chamando a atenção para o elevado índice encontrado nas comunidades de São José (28\%) e Lajeado (25\%). A HbC também apresenta prevalência variável na população brasileira, dependendo da região analisada, e nos povos de origem africana pode chegar a $30 \%$ da população ${ }^{34}$.

Quanto à distribuição das hemoglobinas variantes em relação ao gênero, a teoria mais aceita na genética das hemoglobinas é a típica mendeliana. Os genes que controlam a síntese da cadeia beta das hemoglobinas A, S e C são alélicos e herdados como autossômicos codominantes, sem nenhuma diferença na prevalência dos genótipos entre os sexos. No entanto, alguns estudos sugerem que há um efeito materno, principalmente para anemia falciforme, havendo o nascimento de mais mulheres do que homens com essa informação genética ${ }^{35,36}$. Neste estudo, foram identificadas três pessoas do gênero feminino para uma do gênero masculino com HbSS (3:1), e 19 pessoas do gênero feminino para 28 do gênero masculino com traço para $\mathrm{HbS}(0,7: 1)$ (Tabela 3). Somados o número de pessoas do gênero feminino (22) e masculino (29) com a informação genética para HbS, obteve-se uma relação de 0,8:1. Desse modo, não se pode sugerir o efeito materno para os resultados aqui apresentados.

Quando analisada a distribuição do traço falciforme por faixa etária, foi identificado um importante quantitativo de pessoas em idade reprodutiva (Tabela 3), se considerada a idade de 13 a 46 anos para as mulheres ${ }^{37}$ e acima de 13 anos para os homens ${ }^{38}$. Das comunidades quilombolas estudadas, somente a Chapada de Natividade é urbana; as demais estão em ambiente rural e são mais isoladas 
geográfica e culturalmente. Os casamentos e/ou a formação das famílias comumente ocorrem entre os indivíduos da própria comunidade, o que aumenta a possibilidade de interação entre as hemoglobinas variantes e, consequentemente, do nascimento de pessoas com DF.

Historicamente, a teoria mais aceita para a mutação da $\mathrm{HbS}$ e da HbC foi a de uma proteção seletiva dos heterozigotos contra os efeitos letais da infecção causada por Plasmodium spp., que foi transferida geneticamente para outras gerações ${ }^{1,39}$. Os estudos vêm demonstrando a importância dessas hemoglobinas variantes no controle das manifestações clínicas da malária. $\bigcirc$ trabalho de Joishy et al. ${ }^{40}$ mostrou que a frequência de malária causada pelo Plasmodium falciparum, na Índia, foi menor em indivíduos com traço falciforme do que em indivíduos normais (HbAA). Já Modiano et al. ${ }^{41}$ observaram redução das manifestações clínicas da malária em indivíduos heterozigotos e homozigotos para a $\mathrm{HbC}$. Outros estudos demonstraram que pessoas com traço falciforme apresentam limitação para a expansão da infecção desencadeada pelo parasita, o que ocorre devido ao aumento da resposta imune contra - Plasmodium ${ }^{4,42}$. Verra et al. ${ }^{43}$ demonstraram que tanto a $\mathrm{HbS}$ como a $\mathrm{HbC}$ afetam o desenvolvimento precoce de imunidade naturalmente adquirida contra a malária. Para esses autores, a reatividade imunológica melhorada nas pessoas com essas hemoglobinas variantes sustenta a hipótese de que a proteção contra a malária pode ser, pelo menos, parcialmente mediada por imunidade adquirida contra a doença.

No Tocantins, estudos relataram casos de malária com as espécies Plasmodium vivax, P. falciparum e Plasmodium malariae ${ }^{44,45}$. A presença de pessoas $\mathrm{HbS}$ e $\mathrm{HbC}$, demonstrada neste trabalho, pode ser um fator limitante para a proliferação da malária em algumas regiões desse Estado. Um estudo realizado por Parise et al. ${ }^{44}$ mostrou que, no Tocantins, os municípios com maior frequência de casos de malária estão às margens do Rio Araguaia e associa isso a fatores como a proximidade das praias fluviais e da vegetação.

As comunidades quilombolas ao norte do Tocantins, onde estão os municípios citados no trabalho de Parise et al. ${ }^{44}$, não foram investigadas quanto ao diagnóstico das hemoglobinas variantes. No entanto, na região do Jalapão, no município de Mateiros, estão as comunidades Quilombolas dos Rios, Mumbuca e Carrapato, Formiga e Ambrósio, onde 7,3\% da população das três comunidades apresentaram hemoglobinas variantes ( $\mathrm{HbS}$ e $\mathrm{HbC}$; resultado deste trabalho). Para o Jalapão, o estudo de Parise et al. ${ }^{44}$ descreveu um índice muito baixo de casos de malária se comparado com outras regiões do Estado (14 casos de malária em seis anos). Assim, a presença dessas hemoglobinas pode ser um fator limitante para a manifestação da malária, hipótese que deverá ser investigada em estudos futuros.
Cabe destacar que as pessoas que participaram deste estudo foram diagnosticadas e passaram a conhecer a condição das suas hemoglobinas. No entanto, 0 presente trabalho conseguiu alcançar somente $65 \%$ da população das famílias investigadas, além de haver outras comunidades que ainda não foram pesquisadas, o que sinaliza para a possibilidade de mais portadores de hemoglobinas variantes. Portanto, fica evidente a importância de trabalhos que continuem a investigar as hemoglobinas hereditárias, além do aconselhamento genético e do diagnóstico neonatal.

\section{CONCLUSÃO}

Apesar dos resultados obtidos para o traço de HbS e $\mathrm{HbC}$ estarem dentro do esperado para a Região Norte do Brasil, destaca-se a elevada prevalência desses em algumas das comunidades quilombolas, - que sinaliza risco iminente para 0 aumento da incidência da DF. Dentre as 14 comunidades estudadas, cinco merecem especial atenção: Chapada de Natividade, Lajeado, Quilombolas dos Rios, São Joaquim e São José, algumas isoladas geograficamente, com pouco acesso à informação e grande possibilidade de futuros nascimentos de pessoas com DF.

É importante considerar que a elevada prevalência da DF aqui relatada não reflete a realidade no Estado. No decorrer deste estudo, foram identificadas pessoas com manifestações clínicas semelhantes às observadas na DF, que não autorizaram sua participação na pesquisa. Além disso, existe a possibilidade de pessoas com DF de origem quilombola não habitarem mais as áreas rurais da sua comunidade, mas os centros urbanos, onde o acesso ao tratamento médico é facilitado.

Diante do exposto, fica evidente a importância de trabalhos que continuem a investigar as hemoglobinas que ocasionam anemias hereditárias e a necessidade de estudos que relacionem essas hemoglobinas com outras doenças, a fim de compreender melhor suas relações. Por fim, os resultados aqui apresentados poderão nortear as políticas públicas voltadas para a DF e, também, reforçam a forte influência africana na constituição da população tocantinense.

\section{AGRADECIMENTOS}

À Dra. Luciana de Oliveira Almeida e à Vélma de Souza Santos Inácio (Associação de Falcêmicos do Estado do Tocantins - AFETO), pelo apoio técnico científico.

\section{APOIO FINANCEIRO}

Secretaria de Desenvolvimento Econômico, Ciência, Tecnologia e Inovação do Estado do Tocantins, edital PPSUS convênio 700.612/08; e Universidade Federal do Tocantins, pelas bolsas concedidas. 


\section{REFERÊNCIAS}

1 Livingstone FB. Hemoglobin history in west Africa. Hum Biol. 1976 Sep;48(3):487-500.

2 Nagel RL, Fabry ME, Pagnier J, Zohoun I, Wajcman $H$, Baudin V, et al. Hematologically and genetically distinct forms of sickle cell anemia in Africa - the Senegal type and the Benin type. $N$ Engl J Med. 1985 Apr;312(14):880-4

3 Rihet P, Flori L, Tall F, Traoré AS, Fumoux F. Hemoglobin $C$ is associated with reduced Plasmodium falciparum parasitemia and low risk of mild malaria attack. Hum Mol Genet. 2004 Jan;13(1):1-6.

4 Kreuels B, Kreuzberg C, Kobbe R, Ayim-Akonor M, Apiah-Thompson P, Thompson B, et al. Differing effects of $\mathrm{HbS}$ and $\mathrm{HbC}$ traits on uncomplicated falciparum malaria, anemia, and child growth. Blood. 2010 Jun;15(2):4551-8.

5 Jesus JA. Doença falciforme no Brasil. Gaz Med Bahia. 2010 ago-out;80(3):8-9.

6 Naoum PC, Bonini-Domingos CRB. Doença falciforme no Brasil: origem, genótipos, haplótipos e distribuição geográfica. J Bras Patol. 1997 jul-set;33(3): 145-53.

7 Silva-Filho G, Lopes MAO, coordenadores. Fragmentos de diásporas africanas no Brasil: sociedade, escravidão, cultura e religiosidades. São José: Premier; 2011.254 p.

8 Ministério da Saúde (BR). Secretaria de Atenção à Saúde. Departamento de Atenção Especializada. Doença falciforme: condutas básicas para tratamento. Brasília: Ministério da Saúde; 2013

9 Ashley-Koch A, Yang Q, Olney RS. Sickle hemoglobin $(\mathrm{Hb} S)$ allele and sickle cell disease: a HuGE review. Am J Genet. 2000 May;151(9): 839-45.

10 Zago MA, Pinto ACS. Fisiopatologia das doenças falciformes: da mutação genética à insuficiência de múltiplos órgãos. Rev Bras Hematol Hemoter. 2007 jul-set;29(3):207-14.

11 Charache S, Conley CL, Waugh DF, Ugoretz RJ, Spurrell JR. Pathogenesis of hemolytic anemia in homozygous hemoglobin C disease. J Clin Invest. 1967 Nov;46(1 1):1795-811.

12 Bonini-Domingos CR. As hemoglobinopatias e a diversidade genética da população brasileira. Rev Bras Hematol Hemoter. 2009;31 (6):401.

13 Harkness DR. Sickle cell trait revisited. Am J Med. 1989 Sep;87(3N):30N-34N.

14 Cançado RD, Jesus JA. A doença falciforme no Brasil. Rev Bras Hematol Hemoter. 2007 jul-set;29(3):203-6.
15 Naoum PC. Interferentes eritrocitários e ambientais na anemia falciforme. Rev Bras Hematol Hemoter. 2000 jan-abr;22(1):5-22.

16 Felix AA, Souza HM, Ribeiro SBF. Aspectos epidemiológicos e sociais da doença falciforme. Rev Bras Hematol Hemoter. 2010 jul-set;32(3):203-8.

17 Murao M, Ferraz MHC. Traço falciforme heterozigose para hemoglobina S. Rev Bras Hematol Hemoter. 2007 jul-set;29(3):223-5.

18 Ducatti RP, Teixeira AEA, Galão HA, BoniniDomingos CR, Fett-Conte AC. Investigação de hemoglobinopatias em sangue de cordão umbilical de recém-nascidos do Hospital de Base de São José do Rio Preto. Rev Bras Hematol Hemoter. 2011 jan-abr;23(1):23-9.

19 Aigner CP, Sandrini F, Duarte EG, Andrade MP, Largura MA, Largura A. Estudo do perfil de hemoglobinas em 9.189 testes realizados no Álvaro Centro de Análises e Pesquisas Clínicas. Rev Bras Anal Clin. 2006 jun;38(2):107-9.

20 Lidani KCF, Barros RF, Bovo F. Relationship between the prevalence of hemoglobin $S$ and the ethnic background of blood donors in Paraná state. J Bras Patol Med Lab. 2015 Jul-Aug;51 (4):212-7.

21 Amorim MM, Tomazi L, Silva RAA, Gestinari RS, Figueiredo TB. Avaliação das condições habitacionais e de saúde da comunidade quilombola Boqueirão, Bahia, Brasil. Bioscienc J. 2013 jul-ago;29(4):1049-57.

22 Souza LO, Teles AF, Oliveira RJ, Lopes MAO, Souza IA, Inácio VSS, et al. Triagem das hemoglobinas $\mathrm{S}$ e $\mathrm{C}$ e a influência das condições sociais na sua distribuição: um estudo em quatro comunidades quilombolas do Estado do Tocantins. Saude Soc. 2013 out-dez;22(4):1236-46.

23 Andrade SP, Teles AF, Souza LO, Silva LC, Oliveia RJ, Santos MG, et al. A distribuição da hemoglobina $S$ em três comunidades quilombolas do estado do Tocantins-Brasil. Scient Amaz. 2015 jan-abr;4(1):10-20.

24 Fundação Cultural Palmares. Quadro geral de comunidades remanescentes de quilombos (CRQs) [Internet]. Brasília: FCP; 2014 [citado 2015 jun 22]. Disponível em: http://www.palmares.gov.br/ wp-content/uploads/crqs/lista-das-crqs-certificadasate-23-02-2015.pdf.

25 Bonini-Domingos CR. Metodologias laboratoriais para o diagnóstico de hemoglobinopatias e talassemias. São José do Rio Preto: HN; 2006.

26 Brasil. Lei $n^{\circ}$ 8.069, de 13 de julho de 1990. Dispõe sobre o Estatuto da Criança e do Adolescente e dá outras providências [Internet]. Brasília (DF): Casa Civil; 1990 [citado 2014 jun 10]. Disponível em: http://www.planalto.gov.br/ ccivil_03/leis/l8069.htm. 
27 Brasil. Lei $n^{\circ} 12.852$, de 5 de agosto de 2013. Institui o Estatuto da Juventude e dispõe sobre os direitos dos jovens, os princípios e diretrizes das políticas públicas de juventude e o Sistema Nacional de Juventude - SINAJUVE [Internet]. Brasília (DF): Casa Civil; 2013 [citado 2014 jun 12]. Disponível em: http://www.planalto. gov.br/ccivil_03/_Ato2011-2014/2013/Lei/ L12852.htm.

28 Brasil. Lei $n^{\circ} 10.741$, de 1 de outubro de 2003. Dispõe sobre o Estatuto do Idoso e dá outras providências [Internet]. Brasília (DF): Casa Civil; 2003 [citado 2014 jun 12]. Disponível em: http://www.planalto.gov.br/ccivil_03/leis/2003/ 110.741.htm.

29 Naoum PC. Sickle cell disease: from the beginning until it was recognized as a public health disease. Rev Bras Hematol Hemoter. 2011 Feb;33(1):7-11.

30 Paiva e Silva RB, Ramalho AS, Cassorla RMS. A anemia falciforme como problema de Saúde Pública no Brasil. Rev Saude Publica. 1993 fev;27(1):54-8.

31 Loureiro MM, Rozenfeld S. Epidemiologia de internações por doença falciforme no Brasil. Rev Saude Publica. 2005 dez;39(6):943-9.

32 Fleury MK. Haplótipos do cluster da globina beta em pacientes com anemia falciforme no Rio de Janeiro: aspectos clínicos e laboratoriais. Rev Bras Anal Clin. 2007 abr-jun;39(2):89-93.

33 Ministério da Saúde (BR). Secretaria de Atenção à Saúde. Departamento de Atenção Especializada. Manual de educação em saúde: linha de cuidado em doença falciforme. Vol. 2. Brasília: Ministério da Saúde; 2009. (Série A. Normas e manuais técnicos).

34 Pinto EA, Lupinacci FL, Santos GER, organizadores. Doenças falciformes: programa de atenção integral às pessoas com doenças falciformes e outras hemoglobinopatias da cidade de São Paulo. São Paulo: Codepps; 2008.

35 Neel JV. The inheritance of sickle cell anemia. Science. 1949 Jul;110(2846):64-6.
36 Torres FR, Bonini-Domingos CR. Hemoglobinas humanas - hipótese malária ou efeito materno? Rev Bras Hematol Hemoter. 2005 jan-mar;27(1):53-60.

37 Smith S, Pfeifer SM, Collins JA. Diagnosis and management of female infertility. JAMA. 2003 Oct;290(13): 1767-70.

38 Kandeel FR, Koussa VKT, Swerdloff RS. Male sexual function and its disorders: physiology, pathophysiology, clinical investigation, and treatment. Endocr Rev. 2001 Jun;22(3):342-88.

39 Flint J, Harding RM, Boyce AJ, Clegg JB. The population genetics of the hemoglobinopathies. Baillieres Clin Haematol. 1998 Mar; 11(1):1-51.

40 Joishy SK, Hassan K, Lopes M, Lie-Injo LE. Clinical, genetic and fertility studies of Indian with $\beta S$-globin gene and the influence of $\mathrm{Hb} \mathrm{S}$ on Plasmodium falciparum malaria infection. Trans R Soc Trop Med Hyg. 1988 Jul-Aug;82(4):515-9.

41 Modiano D, Luoni G, Sirima BS, Simporé J, Verra $F$, Konaté $A$, et al. Hemoglobin $C$ protects against clinical Plasmodium falciparum malaria. Nature. 2001 Nov;414(6861):305-8.

42 Williams TN, Mwangi TW, Roberts DJ, Alexander ND, Weatherall DJ, Wambua $S$, et al. An immune basis for malaria protection by the sickle cell trait. PLoS Med. 2005 May;2(5):e128.

43 Verra F, Simpore J, Warimwe GM, Tetteh KK, Howard T, Osier FHA, et al. Haemoglobin $\mathrm{C}$ and $\mathrm{S}$ role in acquired immunity against Plasmodium falciparum malaria. PLoS One. 2007 Oct;2(10):e978.

44 Parise ÉV, Araújo GC, Pinheiro RT. Análise espacial e determinação de áreas prioritárias para o controle da malária, no Estado do Tocantins, 2003-2008. Rev Soc Bras Med Trop. 2011 jan-fev; 44(1):63-9.

45 Parise ÉV, Araújo GC, Castro JGD. Aspectos epidemiológicos da malária no estado do Tocantins, Brasil e a origem dos casos período 2003 a 2008. Rev Patol Trop. 2012 out-dez; 41 (4):442-56. 